Chapter 4

\title{
Conservative Management of Mitral Insufficiency - An Alternative Approach
}

\author{
Francisco Gregori Junior \\ Additional information is available at the end of the chapter \\ http://dx.doi.org/10.5772/57114
}

\section{Introduction}

Conservative management of mitral insufficiency is an alternative technique with attractive features. Among the many advocates of valve repair, Alain Carpentier is the best known [1] His techniques include a combination of ring annuloplasty, resection of segments of valve leaflets, and shortening, transposition and sectioning of chordae. However, despite this repertoire of repair maneuvers, replacement of the mitral valve is the most common method used for patients with mitral insufficiency.

Mitral insufficiency (MI), defined as blood regurgitation from the left ventricle to the left atrium through the valve, is a situation predisposing to left ventricular dysfunction, increase of the left atrium, and atrial arrhythmias, regardless of the ethiology. MI is a common feature in rheumatic disease and fibroelastic degeneration. Mitral valve prolapse identified by redundancy of the anterior and/or posterior leaflet, papillary muscle dysfunction, and chordae tendineae elongation or rupture may evolve to mitral insufficiency. Mitral valvuloplasty is an elective procedure for repair of MI, with better results than mitral valve replacement (Akins et al, 1994) [2]

We have employed mitral reconstructive surgery in our Service since 1979 according to Carpentier techniques. We introduced new alternative techniques that have been used in parallel to those traditional procedures.

\subsection{Repair of elongated chordae tendineae}

In 1989 we introduced a new technique for shortening of elongated chordae tendineae [3]. It is particularly suitable for shortening the chordae tendineae in patients in whom the papillary muscles are either thin or deeper than usual. The shortening performed above the anterior 
leaflet of the mitral valve, is quite feasible because of the accessible surgical site and easy quantification of the elongation of the chordae tendineae to be corrected.

Once the elongated chordae were identified, the anterior leaflet is exposed and an orifice about two to three millimeters wide is made near the insertion of the elongated chordae (Fig. 1). After that procedure, the elongated chordae is pulled through the orifice with a nerve tractor or even a thick cotton thread so that the anterior leaflet is towered to an appropriate level, resulting in a satisfactory coaptation of the leaflets. The orifice is sutured with interrupted 5-0 polypropylene sutures. The sutures also fasten the chordae to the atrial surface of the anterior leaflet of the mitral valve.

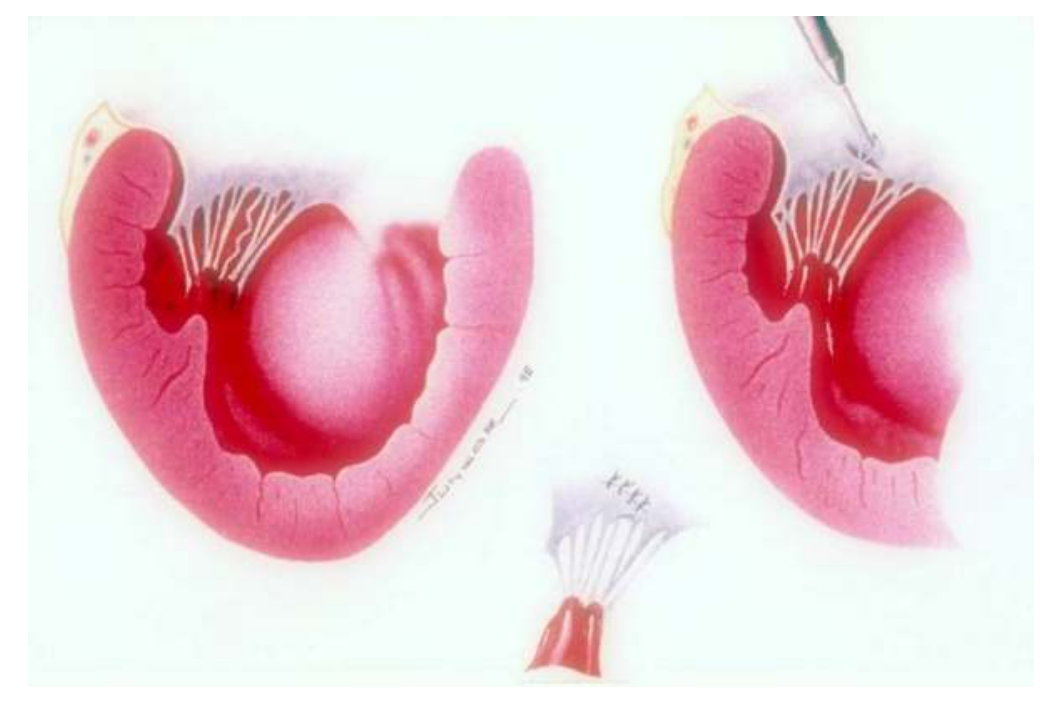

Figure 1. Left - Schematic illustration shows the elongated chorda. Right - Note that the orifice ( 2 to 3 millimeters wide) is near the edge of the leaflet, at a site corresponding to the insertion site of the elongated chordae. Traction of an elongated chordae through the orifice in the anterior leaflet is observed. The orifice in the anterior leaflet is sutured with interrupted 5-0 polypropylene sutures. Lower - The chordae tendineae are fastened to the atrial surface of the mitral valve.

\subsection{Repair of ruptured chordae tendineae}

\subsubsection{Neo chordae construction [4]}

The mitral valve is carefully evaluated and the ruptured or "missing" chordae of the anterior leaflet are located precisely and marked with a suture. Two parallel incisions are made about five to 12 millimeters apart beginning at or near the annulus and carried toward the reference suture up to a point five millimeters from the free border of the leaflet. This strip of tissue thus created is detached beginning near the annulus and is tucked through the slit under the free border, whichever is easier, and brought toward the ventricular cavity. The anterior leaflet is 
then repaired with interrupted 5-0, polypropylene sutures. The strip is next sutured to the anterior papillary muscle with 5-0 polypropylene mattress sutures. (Fig. 2)

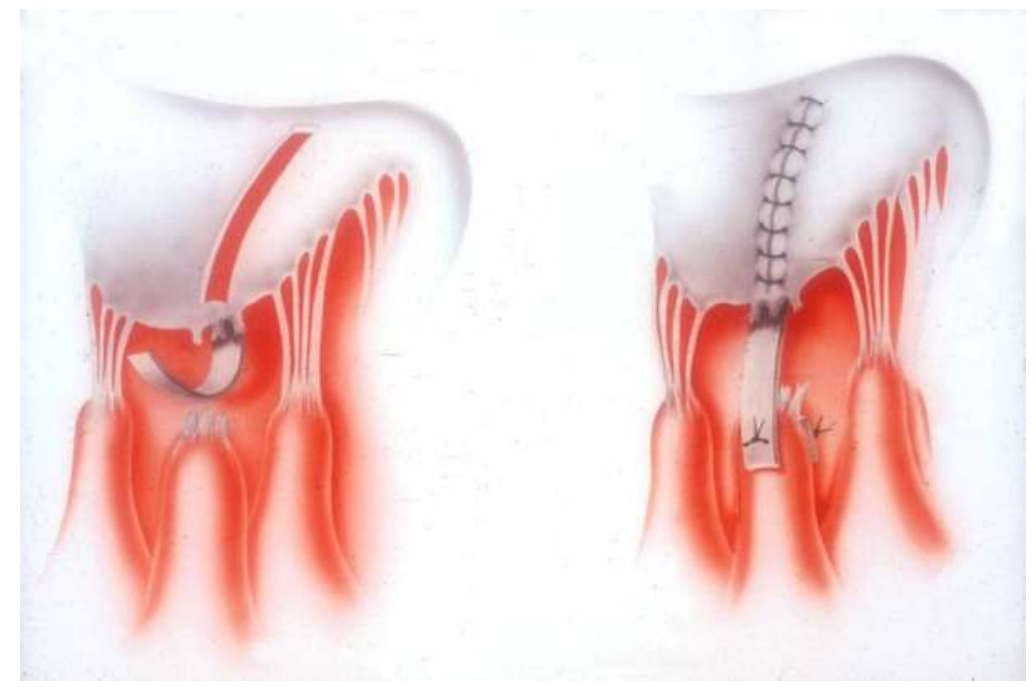

Figure 2. Left - The strip of tissue is tucked through the slit under the edge of the leaflet. Right - The opening in the anterior leaflet is repaired with interrupted 5-0 polypropylene suture and the neochorda is sutured to the anterior papillary muscle with 5-0 mattress sutures.

\section{Partial tricuspid valve transfer [5]}

Supply of chordae for the anterior leaflet of the mitral valve proceeds according to two techniques. The first and more frequent consists of removal of the posterior leaflet of the tricuspid valve of the patient, with all its elements, that is, with chordae and papillary muscle (Fig. 3). The specimen is transferred to the mitral valve by suturing the papillary muscle to that of the mitral valve, corresponding to the ruptured chordae, using one stitche in $U$ anchored on small Dacron pledgets. After attaching the papillary muscle, the donor leaflet is sutured to the anterior leaflet of the mitral valve avoiding too long chordae, that would cause leaflet prolapse and consequent mitral insufficiency. The same care must be taken with regard to the opposite, that is, chordae retraction with unwanted coaptation of the anterior leaflet causing mitral insufficiency (Fig. 4). The grafted leaflet may be sectioned in the middle, remaining with two heads linked to the papillary muscle by a good number of chordae. One of these heads is sutured to the anterior leaflet and the other to the posterior on (Fig. 5). The tricuspid annulus is plicated with 4-0 polypropylene sutures, anchored on Dacron pledgets at the posterior portion, leaving the bicuspid valve, furthermore correcting any eventual functional tricuspid insufficiency. 


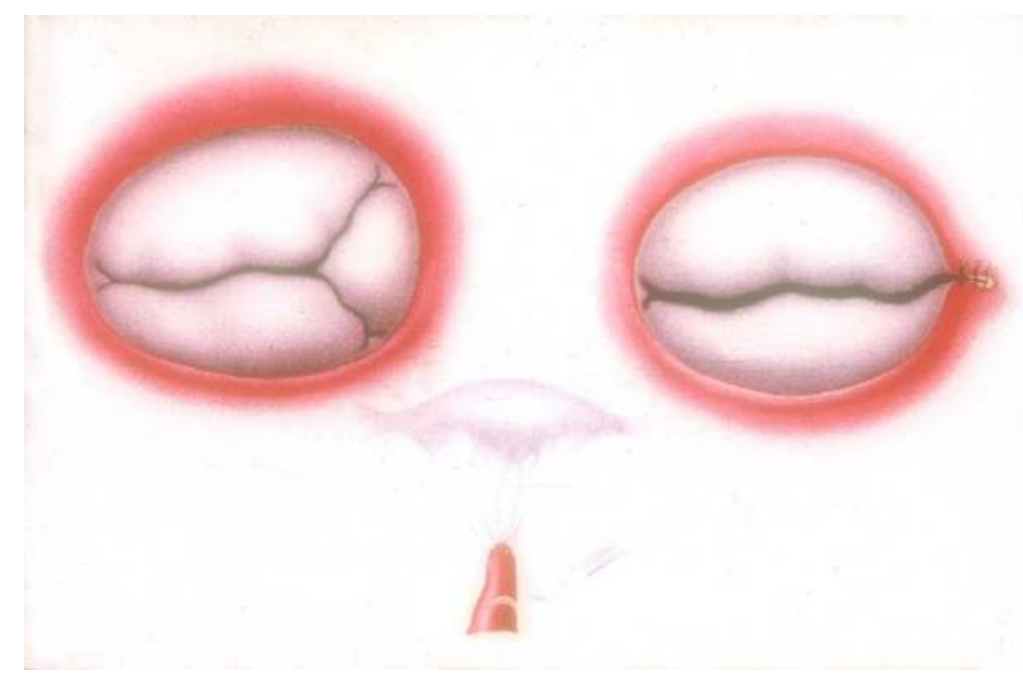

Figure 3. Removal of the posterior leaflet of the tricuspid valve, rendering it bicuspid. The specimen (lower) containing the leaflet, chordae tendineae, and the papillary muscle will be transferred to the mitral valve.

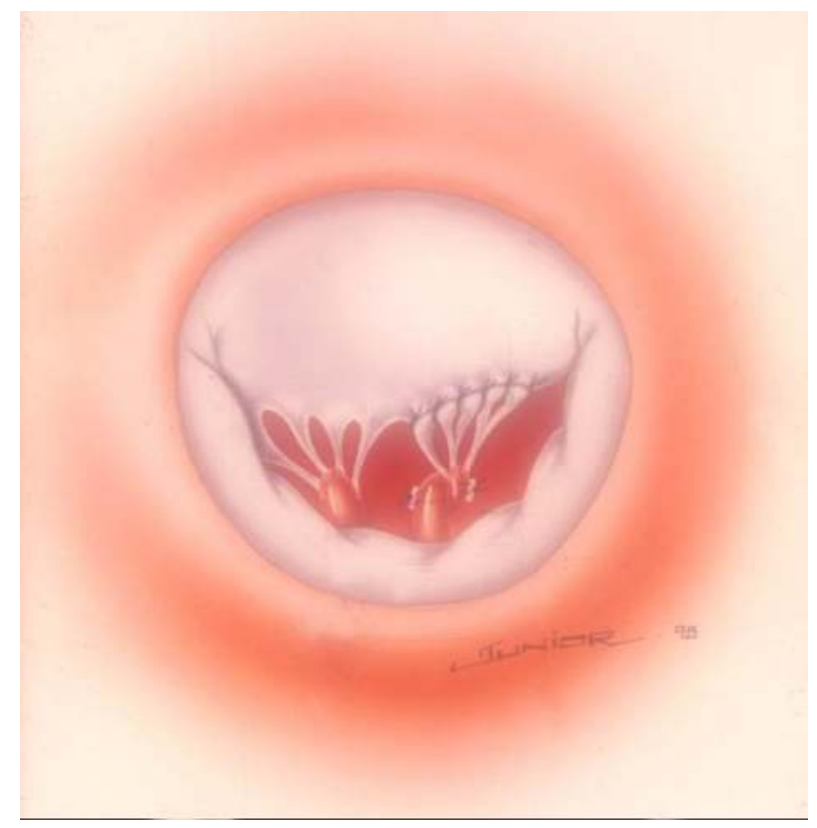

Figure 4. Intraoperative aspect after suture of the graft on the papillary muscle and the anterior leaflet of the mitral valve. 


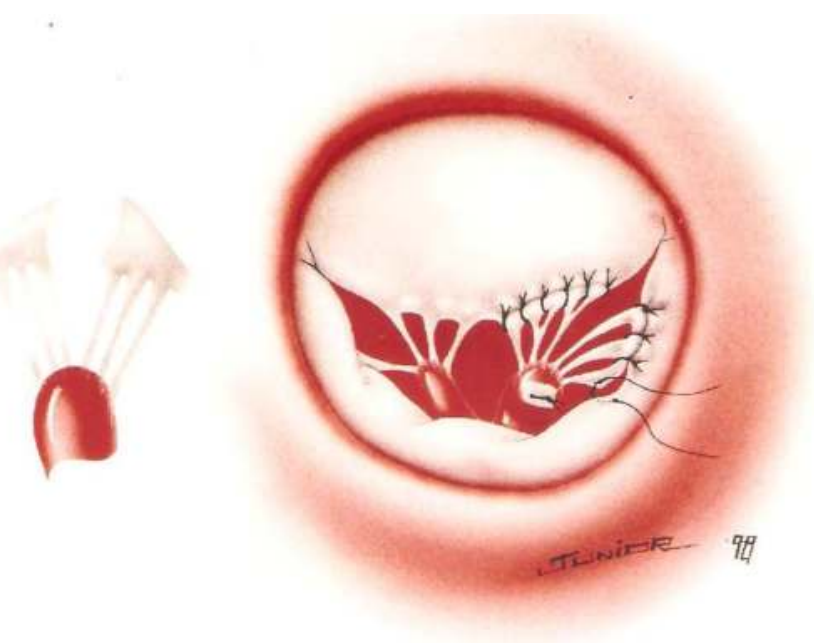

Figure 5. The graft sectioned in the middle remaining with two heads linked to the papillary muscle by a good number of chordae. One head is sutured to the anterior leaflet and the other head to the posterior on.

The second technique (Fig. 6) a variant of the first, is applied when the posterior leaflet of the tricuspid valve, or even the corresponding papillary muscle, were not anatomically adequate. In these cases, the anterior valve is always more developed, to compensate for the small posterior leaflet. Thus, a triangular patch is removed from the anterior leaflet, with a satisfactory number and texture of chordae, and a wedge of the corresponding papillary muscle, wich is transferred to the mitral valve and sutured, likewise to the already described technique. The tricuspid valve in these cases, remains with its three leaflets with the sectioned edges of the anterior leaflet being joined with separated 5-0 polypropylene stitches.

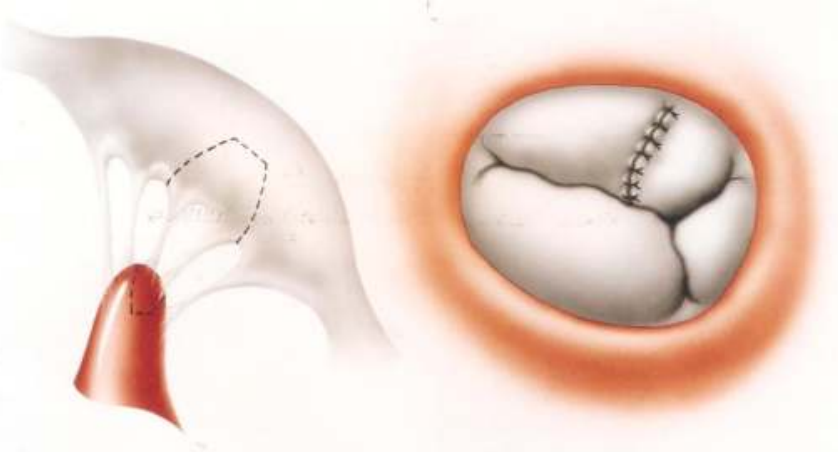

Figure 6. Left - A triangular patch is removed from the anterior leaflet of the tricuspid valve, with a satisfactory number and texture of chordae and a wedge of the papillary muscle which is transferred to the mitral valve. Right - The sectioned edges of the anterior leaflet is joined with separated 5-0 polypropylene stitches. 


\section{Repair of ruptured chordae or thin elongated chordae by premolded bovine pericardium chords [6]}

\subsection{The Braile-Gregori prosthesis}

The Braile-Gregori Prosthesis (Gregori et al, 1994) [9] created for repair of ruptured chordae is fashioned as a monobloc (Braile Biomédica Industria, Comércio e Representações S/A® São Paulo - Brazil) joined at their extremities by two polyester-reinforced strips. The standardized bovine pericardium chordae were two millimeters wide and three millimeters distant from each other (Fig. 7) Standardization of the chordae is confirmed by using measuring instruments ranging in length from 20 to 35 millimeters (Fig. 8) The bovine pericardium is treated with $0.5 \%$ glutaraldehyde, subjected to anticalcification treatment with glutamic acid, and preserved in $4 \%$ formaldehyde solution. Resistance and durability tests showed rupture levels of approximately $15 \mathrm{~kg} / \mathrm{cm} 3$ (Braile et al, 1990) [7]. The chordae length is determined based on the distance from the top of the papillary muscle to the edge of the leaflet in its original nonprolapsed position.

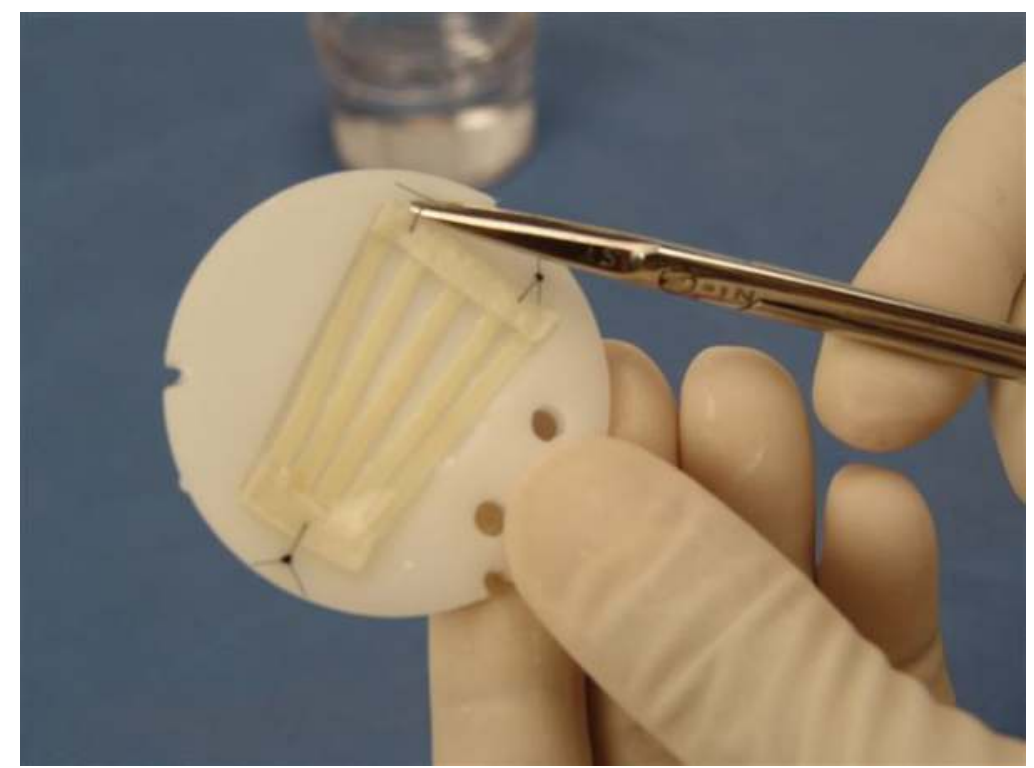

Figure 7. Standardized bovine pericardium chordae.

\subsection{The implantation of the prosthesis}

The implantation procedure began with anchoring of the prosthesis on the top end of the papillary muscle associated with the ruptured chordae, using one or two 5-0 polypropylene threads anchored in a Dacron pad. Subsequently, the other end is attached, using individual 


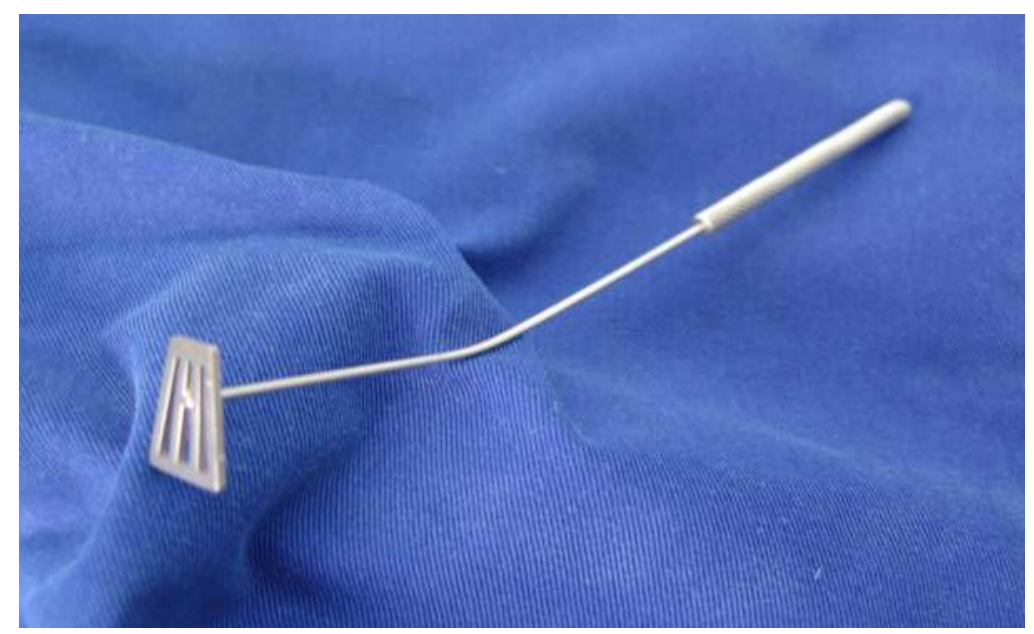

Figure 8. Measuring instruments ranging in length from 20 to $35 \mathrm{~mm}$ are used to confirm standardization of the chordae.

5-0 polypropylene sutures, to the free edge of the affected leaflet (Fig 9). The prosthesis, with five standardized chordae, may be reduced to as few as two chordae, as required.

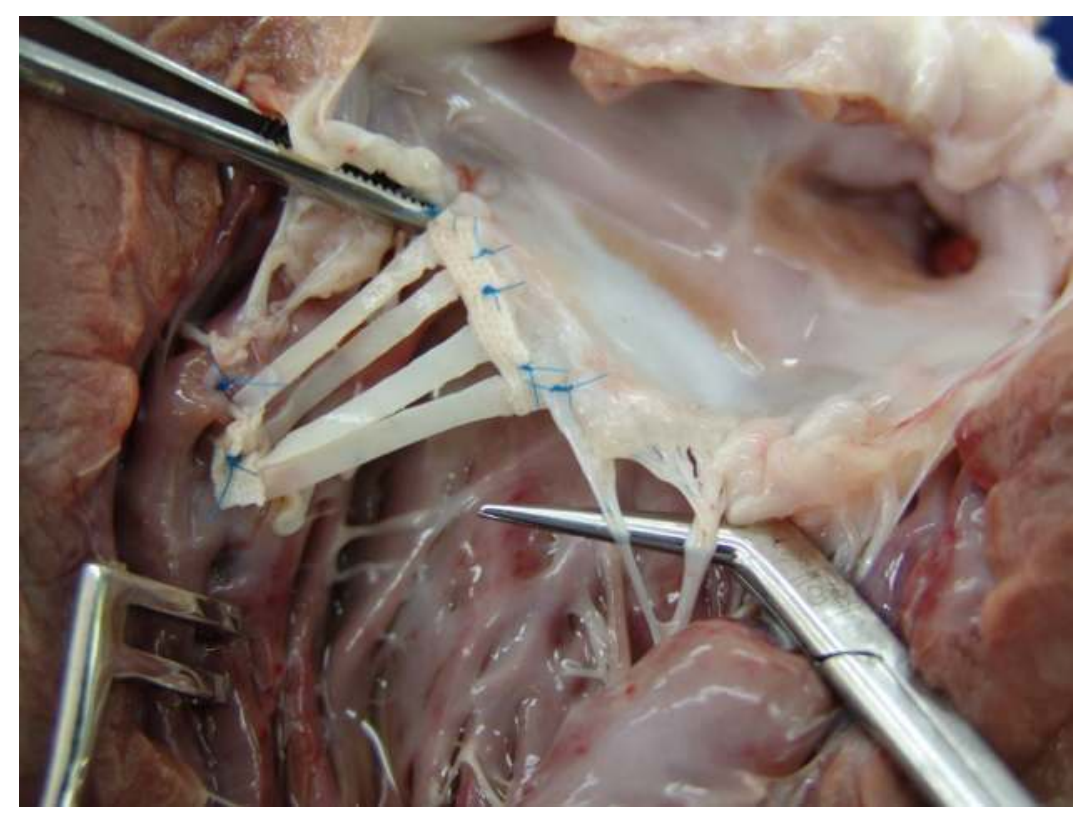

Figure 9. Anatomical aspect of the implantation of the prosthesis in an opened bovine left ventricle. 


\section{Mitral annuloplasty (gregori-braile ring) [9]}

Since the introduction of open valve surgery, annular dilation found in all cases of mitral insufficiency has been treated conservatively. Many Centres, almost simultaneously started correcting mitral insufficiency using plication mitral annuloplasty, a surgical procedure still used today. After having observed anatomic alterations in patients with mitral insufficiency, Carpentier [1] described several techniques for the correction of mitral insufficiency including annuloplasty with a prosthetic ring. Since then, several Centres worldwide have adopted his techniques with excellent results.From 1979 to 1986, we operated on more than 100 patients using Carpentier ring annuloplasty, one-third being under sixteen. Dilation of the anterior side of the mitral annulus between the two fibrous trigonae was demonstrated by Hueb, Jatene and colleagues (2002) [8]. However, it was frequently found posteriorly and to a greater extent posteriorly and next to the postero-medial commissure (Fig. 10). Based on these findings, we developed a semi-circular rigid prosthetic ring (stainless steel wrapped in a thin layer of silicon rubber and covered with Dacron velvet) (Figs.11, 12) with adjustment on its right side, thus correcting the dilation of the posterior side of the mitral ring, next to the postero-medial commissure. The prosthesis corrects the annular dilation (Fig.13) and avoid the late manifestation of mitral stenosis in children and young patients, which is secondary to restraining the normal growth of the mitral ring and observed when closed prosthetic rings are used.
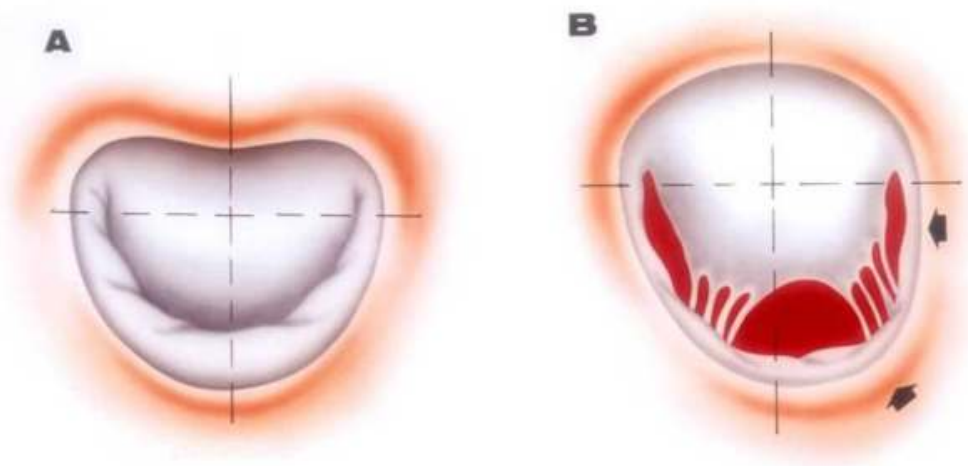

Figure 10. A - Normal mitral valve annulus. B - Posterior dilation of the annulus and to a greater extent next to the postero-medial comissure (arrows). 


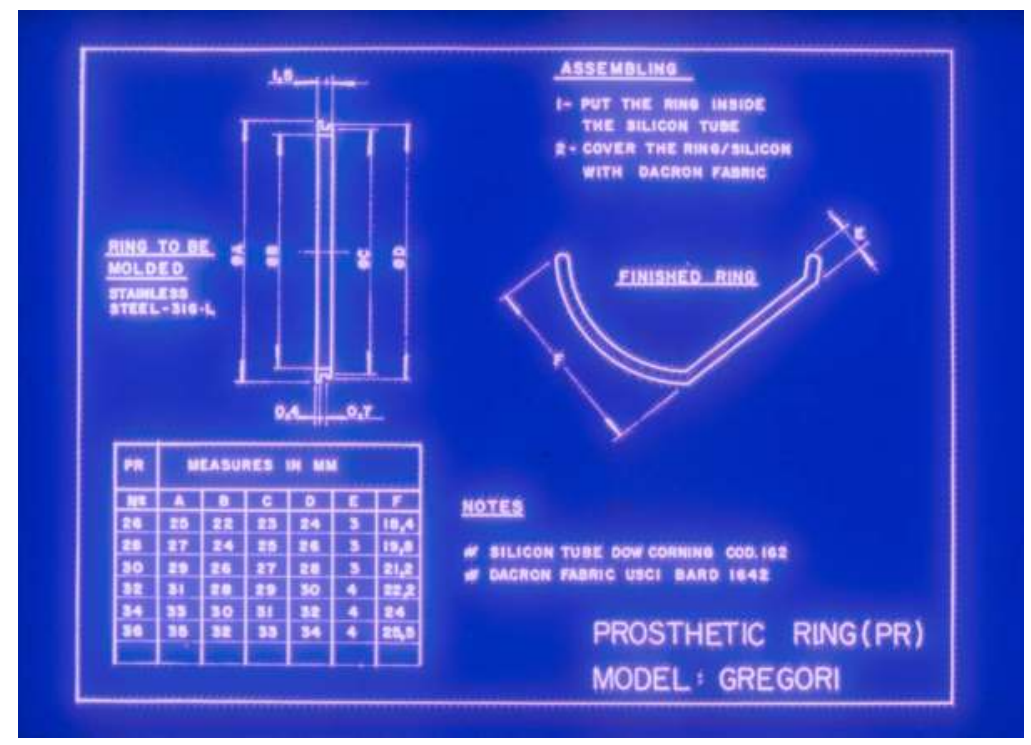

Figure 11. Technical drawing. The assembly of the semi-circular prosthetic ring. Left: measurements of the largest diameter (in millimeters) in A. Right: attention to the rectification on the right that will correct the posterior dilation of the mitral annulus which is greater next to the postero-medial comissure.

Figure 12. Schematic drawing showing the implantation of the Gregori-Braile Ring prosthesis. 


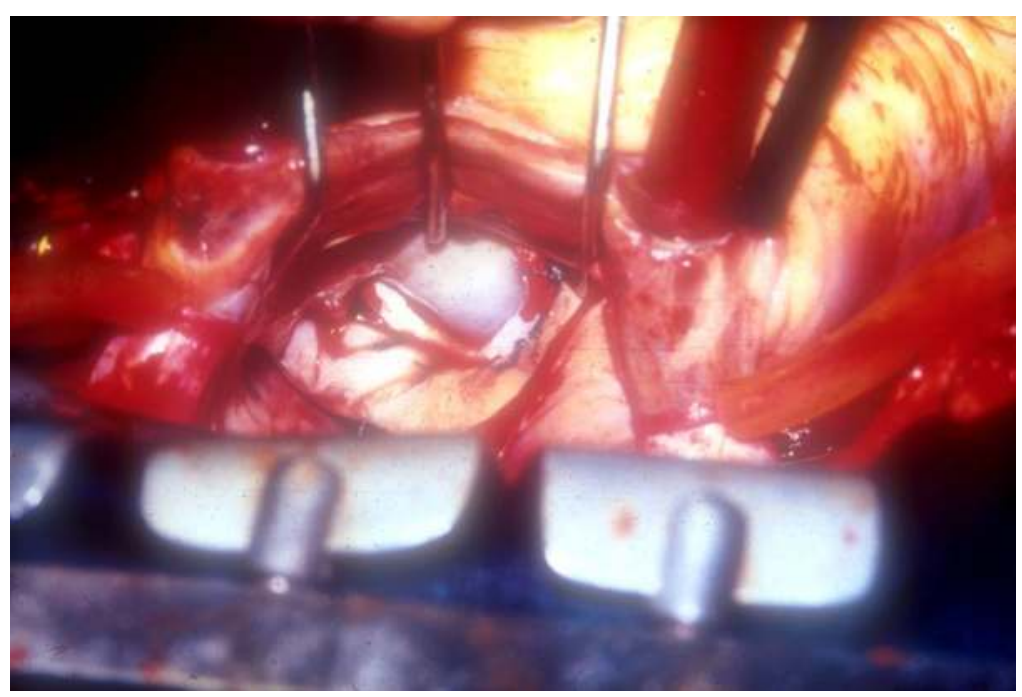

Figure 13. Intraoperative surgical aspect of a mitral annuloplasty by the Gregori-Braile prosthesis ring.

\section{Comments}

The prosthetic ring presented here was first employed in our Centre in October, 1987 [9]. Since then, this prosthesis has been employed in more than 40 Centres in Brazil and other countries. It was developed to correct the dilation of the posterior mitral annulus, resulting in a semicircular shape. In our opinion, the anterior part of the closed ring prosthesis is not only dispensable but also inconvenient in some situations. The posterior ring annuloplasty concept has been adopted by many surgeons as described by Hendren et al [10], Salvador et al [11] and Salati et al [12] who used bovine pericardium strips fixed by glutaraldehyde, and by Braile and colleagues [7] who used berets - also made of bovine pericardium. The mitral valvular system, including the mitral ring grows and develops in children. Implantation of an open prosthesis in children with mitral insufficiency allows for normal growth of the anterior leaflet, which corresponds to the distance between the fibrous trigonae. Any millimeter growth in this area may avoid the late manifestation of mitral stenosis, which happens when closed rings are used in small children. Our own comparative studies in adults have demonstrated the presence of a mitral transvalvar pressure gradient of smaller magnitude in patients with this prosthesis when compared to patients who had the closed ring implanted. The adjustment on the right side of the prosthetic ring was introduced for the correction of small leakage, frequently found next to the postero-medial commissure, since this is the portion of the mitral ring with greater dilation. If required, with the Gregori-Braile ring already implanted, intervention on the subvalvar system is possible with great ease, including the shortening of elongated chordae tendineae and sectioning of retractable chordae. 
The results have been consistent both in adults and children. Machado and Gregori (2005) [13] showed the late evaluation of rheumatic children less than 12 years of age submitted to reconstructive mitral valve surgery with implantation of the Gregori-Braile ring. After 188 months, the survival rate was $82 \%$ and the annual mortality rate, $0.38 \%$. Thirty-one $(72.6 \%)$ patients did not require reoperation and the annual rate of patients who required further surgery was $0.51 \%$.

\section{Author details}

Francisco Gregori Junior

Cardiac Surgery Division, University of Londrina-Paraná School of Medicine, Brazil

\section{References}

[1] Carpentier A, Relland J, Deloche A, Piwinica A, Chauvaud S and Dubost C. Conservative management of the prolapsed mitral valve. Ann Thorac Surg 1978;26:294-302.

[2] Akins CW, Hilgenberg AD, Bukcley MJ, Vlahakes GJ, Torchiana DF, Daggett et al. Mitral valve reconstruction versus replacement for degenerative or ischemic mitral regurgitation. Ann Thorac Surg 1994;58:668-75.

[3] Gregori F Jr, Silva SS, Façanha L, Cordeiro C, Aquino W and Moure O. Preliminary results with a new technique for repairing elongated chordae tendineae of the anterior mitral valve leaflet (Letter). J. Thorac Cardiovasc Surg 1994;107:321-3.

[4] Gregori F Jr, Takeda R, Silva S, Façanha L and Meier MA. A new technique for repair of mitral insufficiency caused by ruptured chordae of the anterior leaflet. J Thorac Cardiovasc Surg 1988;96:765-8.

[5] Gregori F Jr, Cordeiro C, Croti UA, Hayashi SS, Silva SS and Gregori TE. Partial tricuspid valve transfer for repair of mitral insufficiency due to ruptured chordae tendineae. Ann Thorac Surg 1999;68:1686-91

[6] Gregori F Jr, Leal JC and Braile DM. Premolded bovine pericardial chordae for replacement of ruptured or elongated chordae tendineae. Heart Surg Forum. 2010;13:E17-20.

[7] Braile DM, Ardito RV, Pinto GH et al. Plastia mitral. Rev. Bras Cir Cardiovasc 1990;5:86-98

[8] Hueb AC, Jatene FB, Moreira LFP, Pomerantzeff P, Kallás E and Oliveira SA. Ventricular remodeling and mitral valve modifications in dilated cardiomyopathy: New insights from anatomic study. J. Thorac Cardiovasc Surg. 2002;124:1216-24. 
[9] Gregori F Jr., Silva SS, Hayashi SS, Aquino W, Cordeiro C, Silva LR.. Mitral valvuloplasty with a new prosthetic ring. Analysis of the first 105 cases. Eur J. Cardiovasc Surg 1994;8:168-72.

[10] Hendren WG, Nemec JJ, Lytle BW, Loop FD, Taylor PC, Stewart RW et al. Mitral valve repair for ischemic mitral insufficiency. Ann Thorac Surg 1991;52:1246-1255

[11] Salvador L, Rocco F and Lus P. The pericardium reinforced suture annuloplasty: Another tool avaiable for mitral annulus repair? J Cardiovasc Surg 1993;8:79-84

[12] Salati M, Scrofani R, and Santoli C. Posterior pericardial annuloplasty: a physiological correction ?. Eur J Cardiothorac Surg 1991;5:226-9.

[13] Machado VH and Gregori F Jr. Late heart evaluation of children with rheumatic mitral regurgitation submitted to reconstructive surgery with implantation of Gregori's Ring. (in Portuguese) Arq Bras Cardiol. 2005;85:403-11. 